\title{
Polydatin and Atopic Dermatitis in Adults: Clinical Study
}

\section{Giuseppe Maria Izzo ${ }^{1}$ and Giovanni Suffritti ${ }^{2 *}$}

${ }^{1}$ Department of Dermatology, Naples, Italy

${ }^{2}$ Department of Research \& Development, Ghimas, Casalecchio de Reno, Bologna, Italy

"Corresponding author: Giovanni Suffritti, Department of Research \& Development, Ghimas, Via Domenico Cimarosa, 85-40033 Casalecchio di Reno, Bologna, Italy, Tel: +39 051 575353; E-mail: giovanni.suffritti@ghimas.it

Received date: June 07, 2017; Accepted date: July 27, 2017; Published date: July 31, 2017

Copyright: (c) 2017 Giuseppe MZ, et al. This is an open-access article distributed under the terms of the Creative Commons Attribution License, which permits unrestricted use, distribution, and reproduction in any medium, provided the original author and source are credited.

\begin{abstract}
Objective: Verify the clinical efficacy of polydatin applied topically, associated or not with systemic administration of polydatin, on the cutaneous manifestations of moderate to severe atopic dermatitis and check patients' adherence to treatment through a simple evaluation sheet filled in by the investigator and by the patient at baseline and after 7 , 14,30 and 60 days.
\end{abstract}

Methods: Included 128 patients with moderate to severe atopic dermatitis, with lesions on face, on trunk and/or limbs. In 64 of them (age: mean+SEM 35.73+1.03 years - range 18 to 52 years, 33 males and 31 females) a topical cream and a cleanser, both polydatin based were prescribed, while in 64 others (age: mean+SEM 35.75+1.37 years - range 16 to 58 years, 34 males and 30 females) an oral supplement containing 40 mg polydatin was added.

Results: An important reduction of variable degree of lesions and subjective symptoms of atopic dermatitis, such as itching, erythema, xerosis, lichenification of the skin and eczematous lesions has been observed.

Conclusion: The use of topical cream and cleanser associated with oral administration of polydatin tablets restores the function of the skin barrier, counteracts the action of irritants or allergens, reduces the local inflammatory response, and eliminates the itching and scratching.

Keywords: Polydatin; Atopic dermatitis; Topical therapy

\section{Introduction}

In Italy the information and the collection of epidemiological data on atopic dermatitis are still insufficient, especially in those with severe form, representing a minority. To understand the extent of the problems caused by atopic dermatitis is worth taking into consideration in an American survey conducted in more than 500 adult patients with moderate to severe disease.

In over $50 \%$ of cases, the disease has had a negative impact on daily life. The majority of cases have changed their lifestyle to manage the disease, such as choosing a career limiting interactions with other people. And again, half of the patients complained that the disease has undermined self-confidence or caused problems to rest. $28 \%$ are anxious because of their atopic dermatitis, while $23 \%$ of patients are said to be depressed (in some cases the patient comes to attempt suicide). Finally, $20 \%$ confessed that the disease had had negative effects on the ability to maintain their jobs.

Unfortunately, the causes of atopic dermatitis have not yet been well understood, and the disease can really affect anyone. It is manifested by rashes and itching that becomes unbearable, causing scratches and lacerations. Bleeding crusts are often formed. The itching then turns into pain.

Those suffering from atopic dermatitis, even in a serious condition, often make mistakes related to the poor knowledge of the disease or, unfortunately, to despair.
Some of these mistakes consist in submitting to frequent showers.

There is excessive washing, without considering that water can dehydrate the skin and can therefore have a negative effect.

After each shower it would be good to hydrate properly. Some patients do not use the right amount of cream or often use harmful products.

For atopic dermatitis the problem is not quantitative but qualitative. And too much use of creams can be a mistake. Often there is an abuse of topical corticosteroids that, if used improperly or for too long, can produce unwanted effects.

Based on the results of the studies of researchers about promising anti-inflammatory properties of polydatin [1], the efficacy of treatment in subjects with moderate to severe atopic dermatitis has been tested in persistent forms whose manifestations were more frequently dry, extremely pruriginous, with thickened and lichenized skin.

\section{Polydatin}

The main biological activities of polydatin, which is the natural precursor of resveratrol, are the following: potent antioxidant activity and many of the biological properties are attributed to this activity $[1,2]$, anti-inflammatory activity, modulating the production of regulatory cytokines and pro-inflammatory cytokines [3-5], potent immunomodulatory effects on immune cells [6-8].

Differing from resveratrol that has a bioavailability of less than $2 \%$, polydatin as natural trans-resveratrol glucoside has active transmembrane absorption, reaching a bioavailability of approximately 
$100 \%$, also entering inside the cell, the intracellular organelles and the nucleus [9-13].

The powerful antioxidant and anti-radical action of polydatin allows treatment of all diseases based on inflammation or that determines an inflammatory response and of all conditions caused by increased oxidative stress.

Polydatin normalizes alterations in gastro-intestinal mucosa, allowing improved absorption of substances supplied with food and reduces inflammatory reactions that often cause and maintain the conditions of upset and annoyance of the gastro-intestinal tract [4]

Preventing the accumulation of cholesterol and fat in the liver, polydatin lowers the blood concentration of triglycerides and LDL cholesterol (low-density lipoprotein), while reducing cardiovascular risk and exerting a cardioprotective effect [1].

Polydatin exerts a potent antifree-radical action at both intracellular and extracellular levels, modulating the chronic inflammatory response in all inflammatory and/or degenerative pathologies [2-5].

Polydatin improves the immune system of the skin, decreases the damage of inflammation, increases cellular repair and the release of $\beta$ defensins, which regulate cellular defences against environmental stress [14-19].

In this open, uncontrolled clinical study performed in a center specialized in dermatology in Naples (Italy) we have evaluated the effectiveness of polydatin both for topical use only and in combination with systemic therapy. All subjects provided written informed consent to treatment, in accordance with the ethical guidelines of the Helsinki declaration and its subsequent amendments.

\section{Methods}

In our study we tested the efficacy of a treatment with polydatin on 128 patients (ranging in age between 18 and 58 years old, 67 males and 61 females) with moderate to severe atopic dermatitis, in the arm (in 28 cases), in the popliteal region (in 16 cases), in the neck (in 27 cases), in the wrists (in 13 cases), in retro-ear level (in 9 cases) and in the face (in 35 cases) (Figure 1).
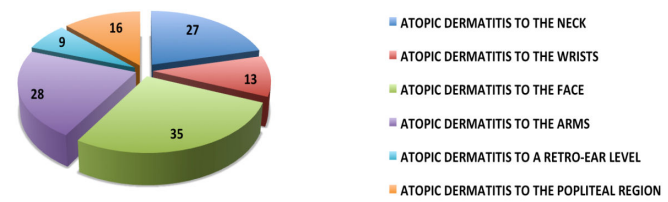

Figure 1: Types of diagnosis of atopic dermatitis.

In the first visit was evaluated, along with a thorough medical history, clinical subjective and objective of atopic dermatitis, with particular reference to itching, erythema, xerosis, lichenification of the skin and eczematous lesions.

Symptoms were assessed with a semi-quantitative rating scale $(0$ absent, 1 - medium grade; 2 - high grade).

Patients were divided according to the treatment in two groups of equal composition:
Topical Polydatin Group - Patients used only a $1.5 \%$ or a $0.8 \%$ polydatin-based cream for two weeks (morning and evening) to be used in relation to the surface of atopic skin lesions, in formulations suitable for subjects who do not tolerate allergenic and hyper-reactive substances such as atopics, accompanied by a cleanser containing polydatin at a concentration of $0.2 \%$ to be used for skin cleansing.

Topical and Systemic Polydatin Group - Patients used the same topical preparations for two weeks and took a two-month-long dietary supplement in $40 \mathrm{mg}$ tablets of polydatin (at the daily dose of two tablets - one in the morning and one in the evening).

The type of treatment - polydatin-based dermocosmetics only or in combination with a polydatin-based dietary supplement - was assigned at random in relation to patient enrolment.

Throughout the duration of the study, all patients have guaranteed not to use on areas with atopic dermatitis other dermocosmetics, other types of soap or cleanser, and other supplements other than the test products.

After 7, 14, 30 and 60 days, patients were reviewed to evaluate the evolution of evaluation parameters.

After 30 and 60 days, both the patient and the doctor expressed the assessment of treatment efficacy (0 - totally ineffective, up to 10 excellent efficacy).

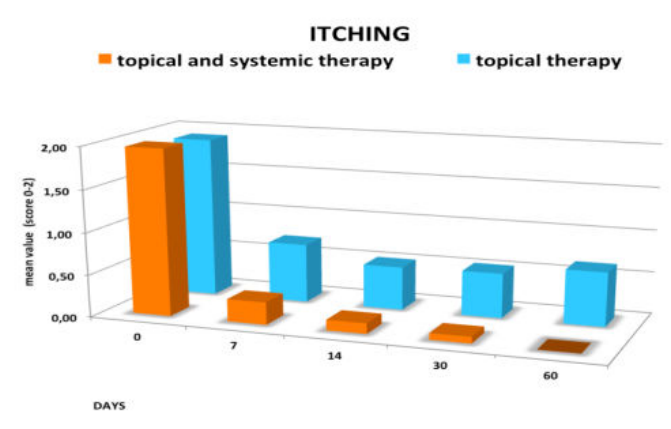

Figure 2: Itching: mean score for each control time in the groups.

\section{Results}

All patients during the study showed a marked reduction in lesions and symptoms, with statistical significance of the variation since the first control after one week.

It should be noted in every way a different performance of the two treatment groups.

In the group of patients who used the only topical product, itching (Figure 2) went down with an average 63\% drop in severity after the first seven days of treatment and $73 \%$ after 14 days. It should be noted, however, that a subsequent reversal of the tendon reversal was observed with a slight increase in itching intensity in 2 patients at 64 after 14 days, in 4 patients in 64 after 30 days and in 8 patients in 64 after 60 days. 
The efficacy of topical and generalized combination therapy with polydatin in the topical and systemic polydatin group is demonstrated by the sharp reduction of itching, the most fearsome subjective symptom of atopic dermatitis, which results or worsens the clinical lesions typical of dermatitis. The decline was $87 \%$ after 7 days, $94 \%$ and $96 \%$ after 14 and 30 days, respectively (only in some cases it was a mild itch, specifically in 8 patients after 14 days and in 5 after 30 days). After 60 days no patient complained of itching.

Redness (Figure 3) has also decreased in all patients treated with POLIDAL cream and cleanser. This parameter has equally showed a biphasic pattern with a drop of up to 14 days and subsequent slight increase; precisely the redness was present in $43 \%$ at 7 days, in $30 \%$ at 14 days, in $33 \%$ at 30 days and in $39 \%$ at 60 days.

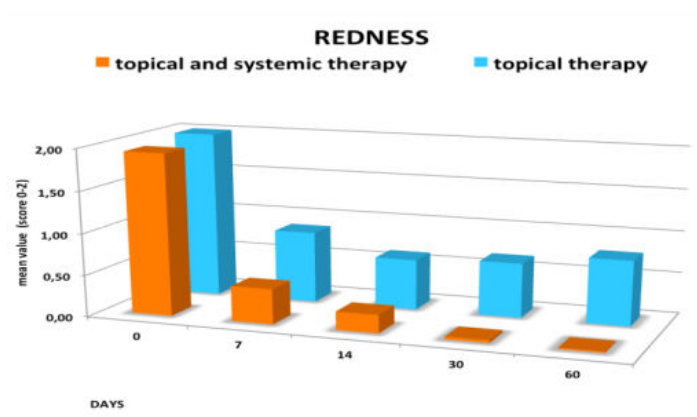

Figure 3: Redness: mean score for each control time in the groups.

As confirmation of what we reported for the previous parameter, in the group of patients who used combined therapy redness decreased rapidly $-78 \%$ at 7 days, $-89 \%$ at 14 days, $-98 \%$ at 30 days, and $-99 \%$ at 60 days (only in 2 cases remained mild erythema after 30 days and in 1 case after 60 days).

The skin xerosis (Figure 4) gradually decreased in the majority of patients who used the only topical products. It is still noticed the biphasic trend with a decrease until the $14^{\text {th }}$ day and subsequent slight increase, and specifically cutaneous xerosis was present in 55\% at 7 days, $36 \%$ at 14 days, $38 \%$ at 30 days and $46 \%$ at 60 days.

In the topical and systemic polydatin group, cutaneous xerosis has undergone a net reduction in intensity, until it disappears almost completely (only one patient had a mild skin xerosis after 60 days).

In all patients in the Polydatin group, skin texture (Figure 5) has improved, appearing much less thickened and lichenized. The decrease in severity was approximately $38 \%$ after the first seven days of treatment and $65 \%$ after 14 days. It should be noted, however, that in the subsequent controls there has been a trend reversal with the stationary level reached.

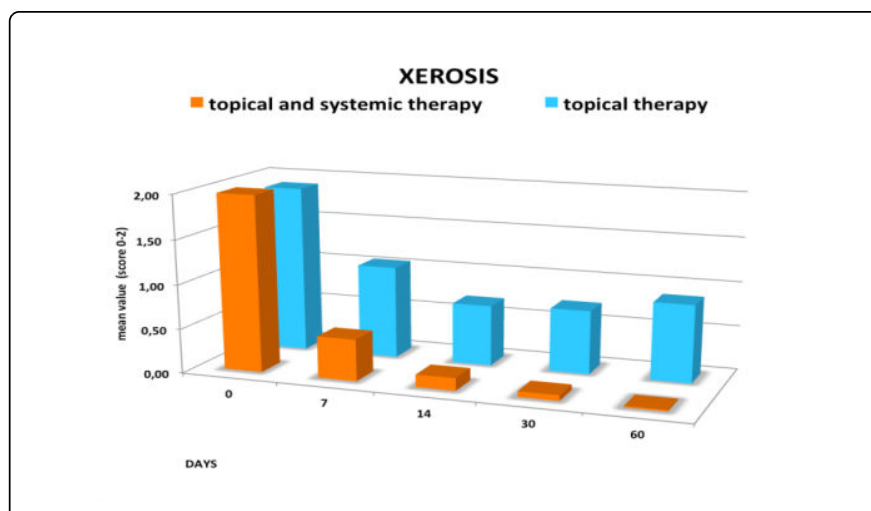

Figure 4: Xerosis: mean score for each control time in the groups.

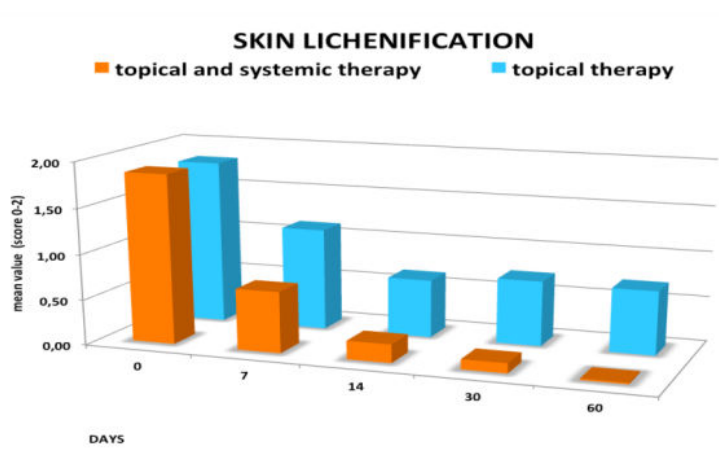

Figure 5: Skin lichenification: mean score for each control time in the groups.

Even skin thickening has gone down in the group of cases treated with POLIDAL topical+POLIDAL tablet combination, with $64 \%$ reduction to 7 days, $89 \%$ at 14 days, $94 \%$ at 30 days and $99 \%$ at 60 days (only in one case remained mild skin lichenification after 60 days).

Eczematous lesions (Figure 6) in patients using the only topical product are gradually improving. Eczematous lesions were present in $75 \%$ at 7 days, $52 \%$ at 14 days, $45 \%$ at 30 days and $39 \%$ at 60 days.

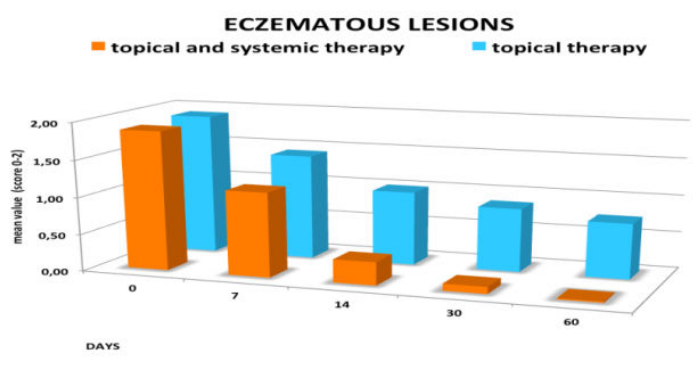

Figure 6: Eczematous lesion: mean score for each control time in the groups.

In the group of patients who used combined therapy, eczematous lesions decreased rapidly: $-39 \%$ at 7 days, $-83 \%$ at 14 days, $-95 \%$ at 30 
Page 4 of 6

days, and $-99 \%$ at 60 days (only one case showed mild eczematous lesions after 60 days).

The variation of all signs and symptoms (Figure 7) confirmed for patients using only the topical product the biphasic efficacy trend, with improvement up to day 14 and subsequent slight worsening.

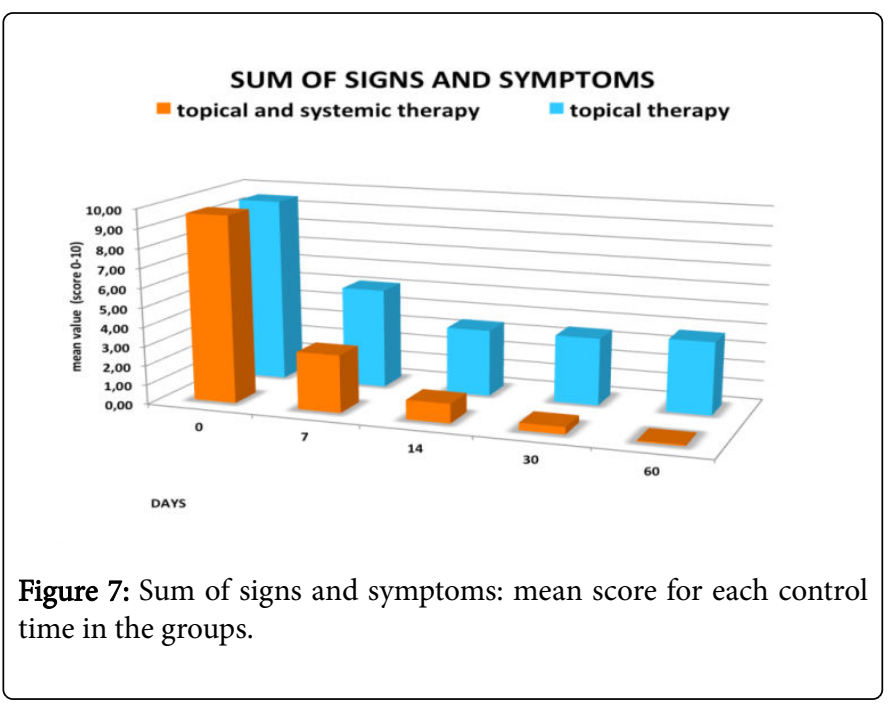

In the group of cases treated with POLIDAL topical+POLIDAL tablet, however, the improvement was gradual and progressive.

\section{Global evaluation}

Physicians and patients expressed a generally similar efficacy judgment after 30 and 60 days, although the patient's evaluation was more critical (Figure 8).

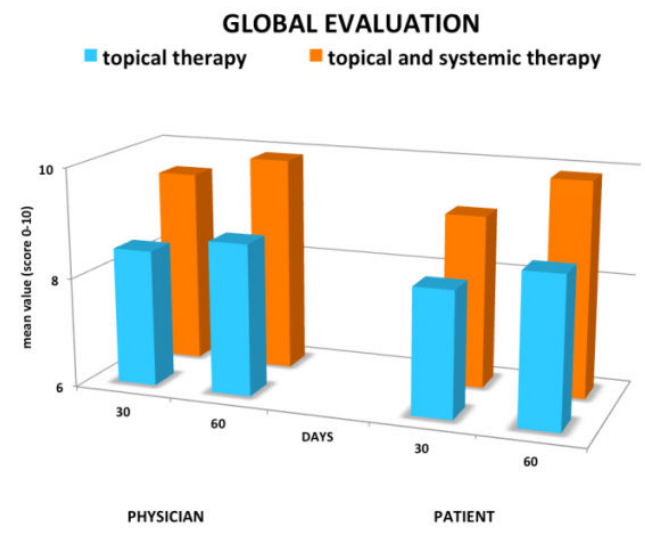

Figure 8: Physician's and patient's global evaluation: mean score after 30 and 60 days in the groups.
For a group of cases treated with only topical therapy both the physician and the patient, after thirty and sixty days of treatment, expressed a very good evaluation rating (scores 8 to 10 ) in $22-25 \%$ of the cases after thirty days and in $28-44 \%$ of cases after sixty days of treatment, good enough (score 4 to 7 ) in $70-73 \%$ of cases after thirty days and in $38-55 \%$ of cases after sixty days of treatment, insufficientpoor (score 0 to 3 ) in $0-2 \%$ of cases after thirty days and in $6-8 \%$ of cases after sixty days of treatment (Figure 9).

\section{GLOBAL EVALUATION}

$\square$ INADEQUATE (score 0-3) $\square$ GOOD (score 4-7) $\square$ EXCELLENT (score 8-10)

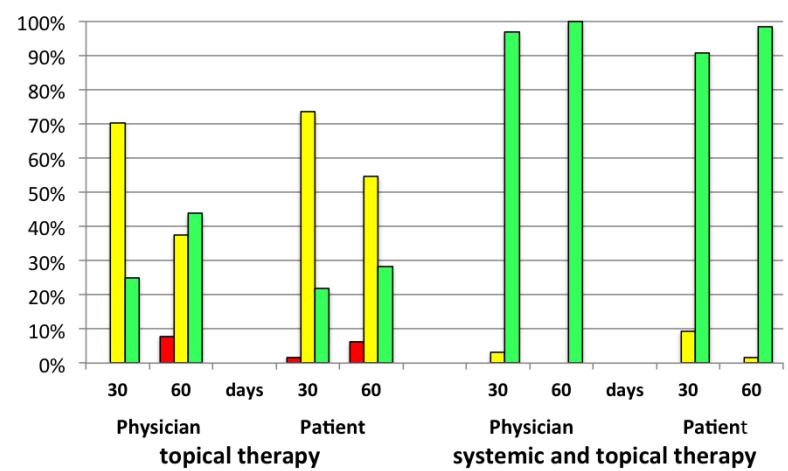

Figure 9: Physician's and patient's global evaluation: frequencies of the scores (inadequate, good, excellent) after 30 and 60 days in the groups.

In the patients treated with POLIDAL topical+POLIDAL tablet, however, overall evaluation was generally excellent.

It should be noted that on average the opinions expressed by physician and patient in the topical treatment group were significantly lower than those expressed in the group of patients undergoing combined POLIDAL topical+POLIDAL tablets.

This confirms that polydatin for topical use is effective in the treatment of atopic dermatitis in the adult, but is necessary to optimize and prolong the result obtained by integrating with the same systemic molecule.

All patients undergoing polydatin treatment, whether topical or systemic, did not report any side effects.

\section{Discussion}

The two groups of patients were comparable by sex, age, weight, height, and type of disease (diagnosis and duration of atopic dermatitis) (Table 1).

\begin{tabular}{|l|l|l|l|}
\hline & Topical treatment & Systemic \& topical treatment & $\begin{array}{l}\text { Statistical } \\
\text { comparison between } \\
\text { groups }\end{array}$ \\
\hline Sex & $33 \mathrm{M}-31 \mathrm{~F}$ & $34 \mathrm{M}-30 \mathrm{~F}$ & N.S. $\left(^{*}\right)$ \\
\hline
\end{tabular}




\begin{tabular}{|c|c|c|c|}
\hline Age (years) & $35,73+1,03$ & $35,75+1,37$ & N.S. $\left({ }^{* *}\right)$ \\
\hline Weight (Kg) & $65,11+1,31$ & $64,56+1,47$ & N.S. $\left({ }^{* *}\right)$ \\
\hline Height $(\mathrm{cm})$ & $165,66+1,20$ & $168,36+1,58$ & N.S. $\left({ }^{* *}\right)$ \\
\hline atopic dermatitis of the arms & 11 cases & 17 cases & \multirow{6}{*}{ N.S. $\left({ }^{*}\right)$} \\
\hline atopic dermatitis of the popliteal region & 8 cases & 8 cases & \\
\hline atopic dermatitis of the neck & 13 cases & 14 cases & \\
\hline atopic dermatitis of the wrists & 8 cases & 5 cases & \\
\hline atopic dermatitis of the retro-ear region & 8 cases & 1 case & \\
\hline atopic dermatitis of the face & 16 cases & 19 cases & \\
\hline Duration of atopic dermatitis (days) & $67,38+6,87$ & $58,11+6,15$ & N.S. $\left({ }^{* *}\right)$ \\
\hline
\end{tabular}

Table 1: Atopic dermatitis in two groups of people.

The data collected in the study show that the intensity of the signs and symptoms of atopic dermatitis has shown a reduction in both the topical therapy group and in the combined systemic and topical treatment. However, there is a clear difference between the two groups. In the group treated with the topical therapy the progression was biphasic (reduction and then stationary or increased), however, in the combined systemic and topical treatment the reduction was rapid and progressive until the almost total disappearance of signs and symptoms.

Specifically, in the topical treatment group, improvement was discontinued just after 14 days at the end of the topical therapy and symptomatology re-emerged albeit in the following 45 days of observation.

All this indicates that in these cases of moderate to severe dermatitis the only topical therapy was not totally resolved, while association with systemic therapy resulted in complete control of the initially present symptomatology. It follows that topical treatment, while showing its effectiveness, especially in cases with more severe disease, must be associated with the systemic system to achieve and maintain good results for a long time.

\section{Conclusion}

The clinical results of our study seem to show that defensins continue to be produced by stressed keratinocytes when treated with polydatin, while under stress keratinocytes they are not able to produce defensins.

In fact, keratinocytes are the main actors of the extremely complex response in the skin that leads to orchestrated recruitment and functions of immune cells, fibroblasts and vascular cells involved in inflammatory responses and wound healing. The epidermal growth factor receptor (EGFR) located on the cell membrane of keratinocytes is widely recognized as a key regulator of many essential processes that underlying skin development, homeostasis, and repair.

The cytoprotective action of polydatin on stressed keratinocytes is documented in vitro not only by the reduction of inflammatory response, but above all by the presence of $\beta$-defensins, which are proteins active against bacteria, fungi and viruses [19] produced by keratinocytes.

Our study clinically confirmed the already in vitro documented effects of polydatin.

The use of POLIDAL cream and cleanser associated with oral administration of POLIDAL tablets restores the function of the skin barrier, counteracts the action of irritants or allergens, reduces the local inflammatory response, and eliminates the itching and scratching, thus blocking the "rash-itch-scratch" vicious cycle.

This study confirms that the application of a polydatin-based cream is effective in signs of atopic dermatitis, even moderately-severe, and that the dual topical and systemic administration is effective and safe support for patients with dermatitis atopic, even in its most serious forms, being able to replace or usefully accompany the other topical and systemic treatment types normally used in the treatment of dermatitis.

Therefore, polydatin is a very interesting molecule that can be utilized both in the dermatological field and in the care and prevention of photo aging.

We do think though those other clinical trials are mandatory to fully assess the possible uses of the molecule.

\section{Acknowledgements}

Editorial support for this article was provided by Research \& Development of Ghimas (Italy).

POLIDAL is marketed by Ghimas S.p.A. (Casalecchio di Reno - BO, Italy) under exclusive license GLURES - Spin-off of Ca' Foscari University of Venice (Italy) and is the result of Italian research, with the contribution of the MIUR (RIC 1009 Decree: 16/07/2007 Legislative Decree No. 297/1999). Polydatin, natural glycoside of resveratrol, is extracted with a patented method in Europe (EP2087894A1) and Italy (0001388133 MISE).

The opinions expressed in the current article are whose of the author. The author is fully responsible for all content, editorial decision and opinions expressed in this article. The author received no 
Citation: Giuseppe MZ, Giovanni S (2017) Polydatin and Atopic Dermatitis in Adults: Clinical Study. J Cosmo Trichol 3: 122. doi:

Page 6 of 6

honoraria or other form of financial support related to the development of this manuscript.

\section{References}

1. Fabris S, Momo F, Ravagnan G, Stevanato R (2008) Antioxidant properties of resveratrol and piceid on lipid peroxidation in micelles and monolamellar liposomes. Biophys Chem 135: 76-83.

2. Cui XY, Kim JH, Zhao X, Chen BQ, Lee BC, et al. (2006) Antioxidative and acute anti-inflammatory effects of Campsis grandiflora flower. J Ethnopharmacol 103: 223-228.

3. Lanzilli G, Cottarelli A, Nicotera G, Guida S, Ravagnan G, et al. (2012) Anti-inflammatory effect of resveratrol and polydatin by in vitro IL-17 modulation. Inflammation 35: 240-248.

4. Yang B, Li JJ, Cao JJ, Yang CB, Liu J, et al. (2013) Polydatin attenuated food allergy via store-operated calcium channels in mast cell. World J Gastroenterol 19: 3980-3989.

5. Yuan M, Li J, Lv J, Mo X, Yang C, et al. (2012) Polydatin (PD) inhibits IgE-mediated passive cutaneous anaphylaxis in mice by stabilizing mast cells through modulating $\mathrm{Ca}^{2}+$ mobilization. Toxicol Appl Pharmacol 264: 462-469.

6. Fuggetta M, Mattivi F (2011) The immunomodulating activities of resveratrol glucosides in humans. Recent Pat Food Nutr Agric 3: 81-90.

7. Fuggetta MP, D'Atri S, Lanzilli G, Tricarico M, Cannavò E, et al. (2004) In vitro antitumour activity of resveratrol in human melanoma cells sensitive or resistant to temozolomide. Melanoma Res 14: 189-196.

8. Jeong ET, Jin MH, Kim MS, Chang YH, Park SG (2010) Inhibition of melanogenesis by piceid isolated from Polygonum cuspidatum. Arch Pharm Res 33: 1331-1338.

9. Du QH, Peng C, Zhang H (2013) Polydatin: a review of pharmacology and pharmacokinetics. Pharm Biol 51: 1347-1354.

10. Boocock DJ, Faust GE, Patel KR, Schinas AM, Brown VA, et al. (2007) Phase I dose escalation pharmacokinetic study in healthy volunteers of resveratrol, a potential cancer chemopreventive agent. Cancer Epidemiol Biomarkers Prev 16: 1246-1252.
11. Lv C, Zhang L, Wang Q, Liu W, Wang C, et al. (2006) Determination of piceid in rat plasma and tissues by high-performance liquid chromatographic method with UV detection. Biomed Chromatogr 20: 1260-1266.

12. Walle T, Hsieh F, DeLegge MH, Oatis JE, Walle UK (2004) High absorption but very low bioavailability of oral resveratrol in humans. Drug Metab Dispos 32: 1377-1382.

13. Henry-Vitrac C, Desmoulière A, Girard D, Mérillon JM, Krisa S (2006) Transport, deglycosylation, and metabolism of trans-piceid by small intestinal epithelial cells. Eur J Nutr 45: 376-382.

14. Ishikawa T, Kanda N, Hau CS, Tada Y, Watanabe S (2009) Histamine induces human beta-defensin-3 production in human keratinocytes. Dermatol Sci 56: 121-127.

15. Kanda N, Watanabe S (2004) Histamine enhances the production of granulocyte-macrophage colony-stimulating factor via protein kinase $\mathrm{Ca}$ and extracellular signal-regulated kinase in human keratinocytes. J Invest Dermatol 122: 863-872.

16. Kanda N, Watanabe S (2007) Histamine enhances the production of human beta-defensin-2 in human keratinocytes. Am J Physiol Cell Physiol 293: C1916-1923.

17. Pastore S, Lulli D, Fidanza P, Potapovich AI, Kostyuk VA, et al. (2012) Plant polyphenols regulate chemokine expression and tissue repair in human keratinocytes through interaction with cytoplasmic and nuclear components of epidermal growth factor receptor system. Antioxid Redox Signal 16: 314-328.

18. Potapovich AI, Lulli D, Fidanza P, Kostyuk VA, De Luca C, et al. (2011) Plant polyphenols differentially modulate inflammatory responses of human keratinocytes by interfering with activation of transcription factors NFkB and AhR and EGFR-ERK pathway. Toxicol Appl Pharmacol 255: 138-149.

19. Ravagnan G, De Filippis A, Cartenì M, De Maria S, Cozza V, et al. (2013) Polydatin, a natural precursor of resveratrol, induces $\beta$-defensin production and reduces inflammatory response. Inflammation 36: 26-34. 